\title{
Papers
}

\section{Quetiapine and rivastigmine and cognitive decline in Alzheimer's disease: randomised double blind placebo controlled trial}

Clive Ballard, Marisa Margallo-Lana, Edmund Juszczak, Simon Douglas, Alan Swann, Alan Thomas, John O’Brien, Anna Everratt, Stuart Sadler, Clare Maddison, Lesley Lee, Carol Bannister, Ruth Elvish, Robin Jacoby

\begin{abstract}
Objectives To determine the respective efficacy of quetiapine and rivastigmine for agitation in people with dementia in institutional care and to evaluate these treatments with respect to change in cognitive performance.

Design Randomised double blind (clinician, patient, outcomes assessor) placebo controlled trial.

Setting Care facilities in the north east of England.

Participants 93 patients with Alzheimer's disease, dementia, and clinically significant agitation.

Intervention Atypical antipsychotic (quetiapine), cholinesterase inhibitor (rivastigmine), or placebo (double dummy).

Main outcome measures Agitation (Cohen-Mansfield agitation inventory) and cognition (severe impairment battery) at baseline and at six weeks and 26 weeks. The primary outcome was agitation inventory at six weeks.

Results 31 patients were randomised to each group, and 80 $(86 \%)$ started treatment (25 rivastigmine, 26 quetiapine, 29 placebo), of whom 71 (89\%) tolerated the maximum protocol dose (22 rivastigmine, 23 quetiapine, 26 placebo). Compared with placebo, neither group showed significant differences in improvement on the agitation inventory either at six weeks or 26 weeks. Fifty six patients scored $>10$ on the severe impairment battery at baseline, $46(82 \%)$ of whom were included in the analysis at six week follow up (14 rivastigmine, 14 quetiapine, 18 placebo). For quetiapine the change in severe impairment battery score from baseline was estimated as an average of -14.6 points ( $95 \%$ confidence interval -25.3 to -4.0) lower (that is, worse) than in the placebo group at six weeks $(\mathrm{P}=0.009)$ and -15.4 points $(-27.0$ to -3.8$)$ lower at 26 weeks $(\mathrm{P}=0.01)$. The corresponding changes with rivastigmine were -3.5 points $(-13.1$ to 6.2$)$ lower at six weeks $(\mathrm{P}=0.5)$ and -7.5 points $(-21.0$ to 6.0$)$ lower at 26 weeks $(\mathrm{P}=0.3)$.

Conclusions Neither quetiapine nor rivastigmine are effective in the treatment of agitation in people with dementia in institutional care. Compared with placebo, quetiapine is associated with significantly greater cognitive decline.
\end{abstract}

\section{Introduction}

People with dementia often experience intrusive neuropsychiatric symptoms. ${ }^{1}$ These are distressing, present major difficulties for caregivers, and accelerate nursing home placement. Antipsychotic drugs are commonly prescribed to many people with dementia (up to $45 \%$ ) in residential or nursing homes, ${ }^{2}$ often for prolonged periods. Antipsychotics have modest efficacy ${ }^{3}$ but are commonly associated with substantial adverse effects such as parkinsonism, tardive dyskinesia, reduced quality of life, and cardiac arrhythmias. More seriously, an increased risk of stroke has been reported in patients taking the two most widely prescribed atypical antipsychotics, risperidone and olanzapine. ${ }^{4}$ Unfortunately, there are no published randomised controlled trials for other atypical antipsychotics in people with dementia, although a published abstract indicates some benefit of quetiapine in those with agitation. ${ }^{5}$ Preliminary evidence indicates that cholinesterase inhibitors may improve agitation, ${ }^{6}$ though this has not been supported by a randomised controlled trial.

One observational study reported a doubling in the rate of cognitive decline in patients with dementia taking antipsychotics. $^{7}$ Although this was a landmark study, the patients were not randomly allocated to treatment and participants were taking typical antipsychotics. The impact on cognition of newer atypical antipsychotics, which have better overall tolerability, was not determined.

We compared quetiapine and rivastigmine with placebo in patients with dementia and agitation in nursing homes in a randomised double blind placebo controlled trial over 26 weeks. Our primary objective was to determine whether either drug was better than placebo for agitation. We also evaluated whether there was a significant difference between the individual active treatments and placebo with respect to cognitive change.

\section{Method}

Participants were all people with dementia living in care facilities in Newcastle. Those with clinically significant agitation reported by a member of staff or a physician were initially assessed for eligibility using the Cohen-Mansfield agitation inventory. ${ }^{8}$ If the total score exceeded 39 and the level of agitation was judged to represent a clinically significant problem, we assessed the patient for inclusion. Our inclusion criteria were a diagnosis of probable or possible Alzheimer's disease ${ }^{9}$; age $>60$; clinically significant agitation for at least six weeks and scores $\geq 4$ on the irritability or aberrant motor behaviour scales of the neuropsychiatric inventory; and no use of antipsychotics or cholinesterase inhibitors for four weeks before entry into the study. We excluded patients known to be sensitive to cholinesterase inhibitors or antipsychotics and those with advanced, severe, progressive, or unstable disease that might interfere with efficacy or put the patient at special risk; disability that might prevent them from completing study procedures; those with severe, unstable, or poorly controlled medical conditions; bradycardia $(<50)$, sick sinus syndrome, or conduction defects; current diagnosis of active uncontrolled peptic ulceration within the past three 
months; and clinically significant urinary obstruction. All participants, or next of kin or other appropriate person if necessary, gave informed consent.

\section{Evaluations}

We assessed all patients before the start of the pharmacological intervention (baseline) and after six and 26 weeks. At 12 weeks participants underwent an additional severe impairment battery ${ }^{10}$ (to facilitate last observation carried forward) because people with behavioural problems commonly refuse cognitive assessment at least once. Assessors were blind to treatment allocation.

\section{Outcomes}

Our primary outcome measure was a validated standardised evaluation of agitation at six weeks. ${ }^{8}$ We used the severe impairment battery for cognitive assessment. This scale was developed ${ }^{10}$ and used in pivotal trials to measure cognitive change in people with severe dementia. General assessments included electrocardiography, full blood count, and evaluation of severity of dementia (functional assessment staging) ${ }^{11}$ at baseline.

\section{Randomisation}

The study statistician randomly assigned patients in equal numbers to active quetiapine plus placebo rivastigmine; placebo quetiapine plus active rivastigmine; or placebo rivastigmine plus placebo quetiapine (double dummy). The allocations were computer generated with block randomisation (block sizes of three and six) with Stata software (release 7.0). The randomising clinician faxed a form to the statistician, who communicated allocation to the pharmacy, ensuring concealment.

We aimed to attain doses of 25-50 mg quetiapine twice a day and 3-6 mg rivastigmine twice a day by week 12 and doses of 50 mg quetiapine twice a day or $\geq 9 \mathrm{mg}$ rivastigmine daily between week 12 and week 26 . There is inadequate evidence for an optimal dose of quetiapine for behavioural symptoms in dementia. An open study indicated efficacy at $100 \mathrm{mg}$ daily, ${ }^{12}$ which accords with clinical experience and good practice principles of using low doses in dementia.

\section{Sample size and analysis}

To detect an average difference of a 6 point (SD 6) change in agitation inventory score from baseline to six weeks between active treatment and placebo with a power of $90 \%$ at the $5 \%$ (two sided) level of significance, we needed a sample size of 23 in each group, assuming similar efficacy of active treatments. These parameters are based on the effect reported for carbamazepine in a similar study. ${ }^{13}$ With allowance for a drop out rate of $25 \%$, we therefore needed 31 patients per treatment group $(n=93)$.

We used SPSS (release 11.5.0) for Windows to manage data and Stata (release 7.0) for analyses. Demographic factors and clinical characteristics were summarised with counts (percentages) for categorical variables, mean (SD) for normally distributed continuous variables, or median (range) for other continuous variables. We restricted comparative analysis to those patients who had at least one assessment after randomisation. As the trial was blinded, knowledge of allocation could not have contributed to drop outs before or after treatment and so exclusion of such patients did not impart bias. Thus we performed a modified intention to treat analysis with all patients with available data being analysed in the groups to which they were allocated. For the agitation inventory score, for everyone who completed the six week evaluation but dropped out thereafter we carried forward the last total score. For the severe impairment battery, we carried forward the last total score from either the six week or supplementary three month assessment.
For the primary analysis, we summarised the change in the agitation inventory score from baseline to six weeks using the mean (SD). To establish the magnitude and direction of the treatment effect, we used analysis of covariance to compare pairs of treatment groups, giving the mean difference (in change in agitation inventory from baseline to six weeks) between groups (plus 95\% confidence intervals) with adjustment for baseline value. We also evaluated the change in the agitation inventory score from baseline to 26 weeks and the changes in severe impairment battery score. To allow us to detect a change we excluded from the analysis those patients whose scores on the severe impairment battery were $<10$ because of the risk that they would cluster unchanged at the lower end of the score range (a "floor effect").

We made no formal adjustment for multiple significance testing. To meet our prespecified main objectives, our principal comparisons were of active treatment versus placebo on the agitation inventory and severe impairment battery at six weeks. We have reported active treatment group comparisons primarily for completeness given the dearth of published data. We also report results at the 26 week follow up to show whether the effects are temporary or sustained.

\section{Results}

Ninety three patients (31 per arm), most with severe dementia (functional assessment staging scores $>5$ ) were randomised between September 2001 and April 2003, of whom 80 (86\%) started treatment (25 rivastigmine, 26 quetiapine, 29 placebo). The 13 other participants either withdrew consent (10) or died (3) before pharmacological treatment (fig 1). Seventy one (89\%) tolerated the maximum protocol dose (22 rivastigmine, 23 quetiapine, 26 placebo). Eighty seven (94\%) completed the agitation inventory assessment at baseline and $80(86 \%)$ did so at six weeks (24 rivastigmine, 27 quetiapine, 29 placebo). Wherever possible we assessed outcomes irrespective of whether a patient commenced treatment.

Demographic characteristics and stage of dementia were similar across the three groups (table 1), but there was a slight imbalance on severe impairment battery at baseline (10 point difference in favour of patients in the placebo group).

\section{Agitation inventory}

There were no significant differences between treatments in the change in agitation inventory scores between baseline and six weeks and baseline and 26 weeks (table 2). Similar numbers across the treatment groups showed an improvement in agitation inventory score from baseline to six weeks (that is, reduction in score) (fig 2 ).

\section{Severe impairment battery}

Fifty six of the $93(60 \%)$ participants scored $>10$ on the severe impairment battery at baseline (20 rivastigmine, 17 quetiapine, 19 placebo). Forty six (82\%) of these patients (14 rivastigmine, 14 quetiapine, 18 placebo) also completed it at six weeks. For these 46 patients, the mean (SD) scores at baseline were 71.6 (24.6) placebo; 66.6 (21.3) quetiapine and 65.1 (22.6) rivastigmine (2.6, 7.2 , and 6.3 points higher, respectively, than the scores at six weeks). This could indicate that those with a poorer cognitive function were more likely to drop out. Closer inspection of baseline scores in those who did not complete a six week assessment, however, showed that a combination of very high and very low scores was evenly distributed throughout the three groups. In contrast, baseline figures were almost identical for participants who did or did not complete the agitation inventory at six weeks. 


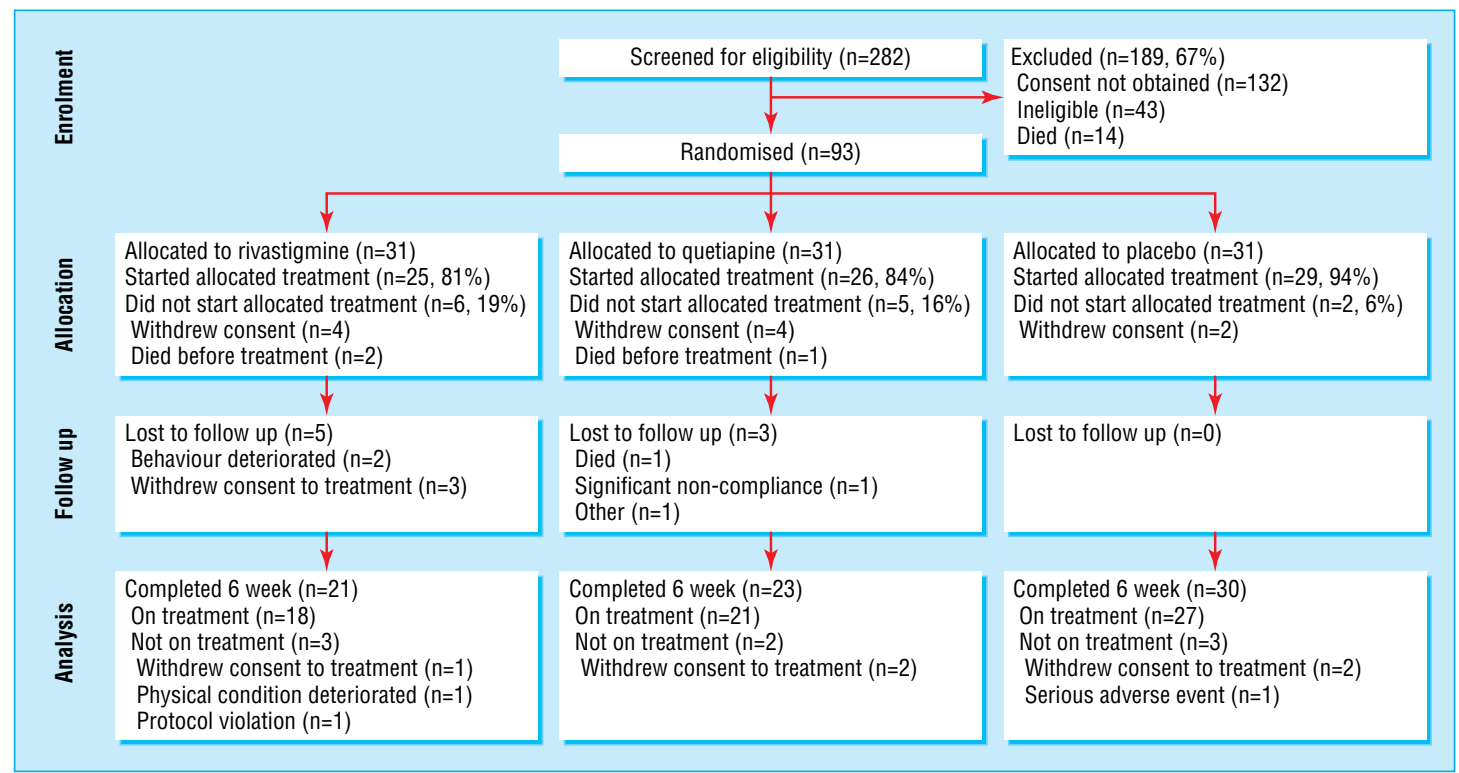

Fig 1 Flow of participants through trial

Patients who received quetiapine experienced, on average, an estimated mean difference in change in severe impairment battery score from baseline of -14.6 points, compared with the placebo treated group at six weeks $(95 \%$ confidence interval -25.3 to $-4.0 ; \mathrm{P}=0.009$ ); indicating a significantly greater deterioration in the quetiapine group (table 2 and fig 3 ). A similar magnitude of difference was evident at 26 weeks $(-15.4,-27.0$ to $-3.8, \mathrm{P}=0.01)$. In contrast, the corresponding comparison of change in score from baseline for rivastigmine with placebo was an average of -3.5 points ( -13.1 to 6.2$)$ lower at six weeks $(\mathrm{P}=0.5)$ and -7.5 points $(-21.0$ to 6.0$)$ lower at 26 weeks $(\mathrm{P}=0.3)$.

\section{Discussion}

Compared with placebo, the atypical antipsychotic drug quetiapine does not result in significant improvement in agitation in patients with dementia and is associated with a greater decline in cognitive function. This result is consistent with data from previous observational and preliminary clinical trials, ${ }^{7}$ highlighting a doubling in the rate of cognitive decline in patients with dementia taking antipsychotics. As we have shown this in a randomised controlled trial, it is unlikely that the differences can be explained by any potential confounding factors. These results have clear implications for clinical practice.

Table 1 Demographic and clinical characteristics and assessments at baseline

\begin{tabular}{|c|c|c|c|c|}
\hline Baseline characteristics & Rivastigmine $(\mathrm{n}=31)$ & Quetiapine $(n=31)$ & Placebo ( $n=31)$ & Overall $(n=93)$ \\
\hline Mean (SD) age (years) & $84.3(7.8)$ & $84.2(8.6)$ & $83.0(6.8)$ & $83.8(7.7)$ \\
\hline No (\%) of women & $23(74.2)$ & $27(87.1)$ & $24(77.4)$ & $74(79.6)$ \\
\hline $\begin{array}{l}\text { No (\%) with significant EPS (M-UPDRS } \\
\geq 8 \text { ) }\end{array}$ & $4(12.9)$ & $4(12.9)$ & $2(6.5)$ & $10(10.8)$ \\
\hline Mean (SD) SIB* (No of patients) & $58.8(27.2)(\mathrm{n}=21)$ & $59.4(30.5)(\mathrm{n}=20)$ & $69.0(28.2)(\mathrm{n}=20)$ & $62.3(28.5)(n=61)$ \\
\hline Mean (SD) CMAI† (No of patients) & $61.1(20.5)(\mathrm{n}=28)$ & $59.1(16.2)(n=28)$ & $56.4(16.6)(n=31)$ & $58.8(17.7)(n=87)$ \\
\hline Median (range) FAST (No of patients) & $6(4-7)(n=26)$ & $6(4-7)(n=28)$ & $6(4-7)(n=30)$ & $6(4-7)(n=84)$ \\
\hline
\end{tabular}

EPS=extrapyramidal symptoms; M-UPDRS=modified unified Parkinson's disease rating scale; SIB=severe impairment battery; CMAI=Cohen-Mansfield agitation inventory; FAST=functional assessment staging.

${ }^{*}$ Range $0-100$. Higher scores mean better performance.

*Range $0-100$. Higher scores mean better performance.
†Range $29-203$. Higher scores mean more agitation. Scores $>40$ usually accepted as clinically significant.

Table 2 Summary of change from baseline at six weeks and 26 weeks for main outcomes plus comparative statistics

\begin{tabular}{|c|c|c|c|c|c|c|}
\hline & \multirow[b]{2}{*}{ Rivastigmine } & \multirow[b]{2}{*}{ Quetiapine } & \multirow[b]{2}{*}{ Placebo } & \multicolumn{3}{|c|}{ Mean difference $(95 \% \mathrm{Cl}), \mathrm{P}$ value } \\
\hline & & & & Rivastigmine v placebo & Quetiapine v placebo & Rivastigmine $v$ quetiapine \\
\hline \multicolumn{7}{|c|}{ Mean (SD) change in CMAI from baseline: } \\
\hline To week 6 & $\begin{array}{c}-5.1(16.3) \\
(n=24)\end{array}$ & $\begin{array}{c}-4.0(15.4) \\
(n=27)\end{array}$ & $\begin{array}{c}-6.2(17.6) \\
(\mathrm{n}=29)\end{array}$ & 4.1 (-4.2 to 12.3$), P=0.3$ & 3.5 (-3.7 to 10.8), $P=0.3$ & 0.29 (-8.0 to 8.6$), P=0.9$ \\
\hline To week 26 & $\begin{array}{c}-10.8(19.9) \\
(n=24)\end{array}$ & $\begin{array}{c}-8.1(12.7) \\
(\mathrm{n}=27)\end{array}$ & $\begin{array}{c}-9.0(16.5) \\
(\mathrm{n}=30)\end{array}$ & $2.2(-5.3$ to 9.7$), P=0.6$ & $2.0(-4.2$ to 8.3$) ; P=0.5$ & -0.52 ( -8.0 to 6.9$), P=0.9$ \\
\hline \multicolumn{7}{|c|}{ Mean (SD) change in SIB from baseline: } \\
\hline To week 6 & $\begin{array}{c}1.8(14.7) \\
(\mathrm{n}=14)\end{array}$ & $\begin{array}{c}-10.5(14.8) \\
(n=14)\end{array}$ & $\begin{array}{c}3.2(15.1) \\
(n=18)\end{array}$ & -3.5 (-13.1 to 6.2), $P=0.5$ & $\begin{array}{cl}-14.6 & (-25.3 \text { to }-4.0) \\
& P=0.009\end{array}$ & 12.0 (0.76 to 23.2), $P=0.04$ \\
\hline To week 26 & $\begin{array}{c}-3.1(20.6) \\
(n=15)\end{array}$ & $\begin{array}{c}-11.3(15.6) \\
(n=15)\end{array}$ & $\begin{array}{c}3.3(17.4) \\
(n=19)\end{array}$ & $-7.5(-21.0$ to 6.0$), P=0.3$ & $-15.4(-27.0$ to -3.8$), P=0.01$ & 8.3 (-5.6 to 22.3), $P=0.2$ \\
\hline
\end{tabular}




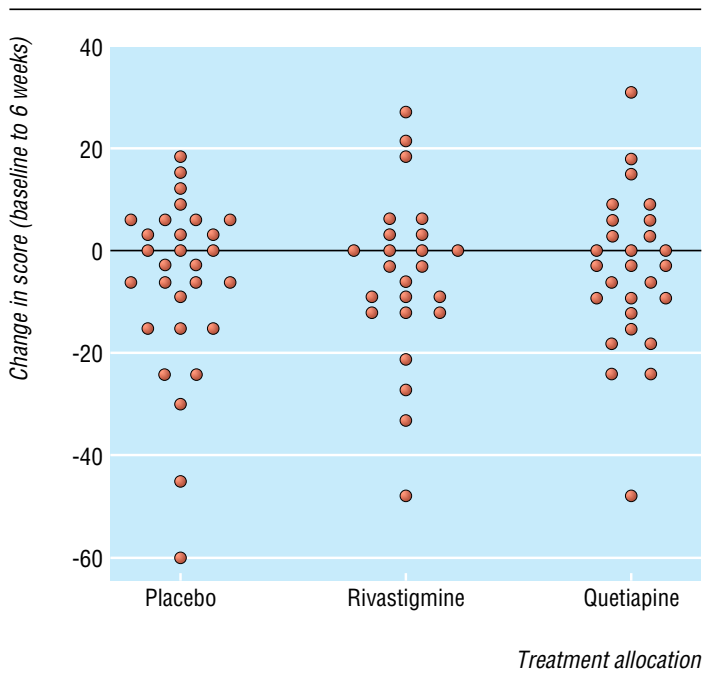

Fig 2 Change in score on Cohen-Mansfield agitation inventory (baseline to six weeks) by treatment group

Even though quetiapine has a relatively more favourable pharmacological profile than other antipsychotic agents, it was still associated with a detrimental impact on cognition. One postulated mechanism is suppression of brain derived neurotrophic factor (BDNF), accelerating the accumulation of the core pathological substrates of Alzheimer's disease. ${ }^{14}$ Another possible mechanism involves antimuscarinic properties. ${ }^{15}$ Although quetiapine has substantially less antimuscarinic activity than some antipsychotics-such as olanzapine, clozapine, and thioridazine-it does have $50 \%$ of the antimuscarinic activity of chlorpromazine and more than twice that of risperidone. ${ }^{16}$ The mechanism may be unrelated to any of these effects but mediated through an impact on other trophic factors. Our findings are contrary to preliminary reports that indicated potential cognitive benefits of quetiapine in people with schizophrenia, ${ }^{17}$ although the mechanisms of cognitive impairment in the two conditions are completely different.

Patients in the rivastigmine group did not experience any significant improvement in agitation nor did they seem to experience a significant decline in cognitive function compared with patients in the placebo group. Further larger studies are

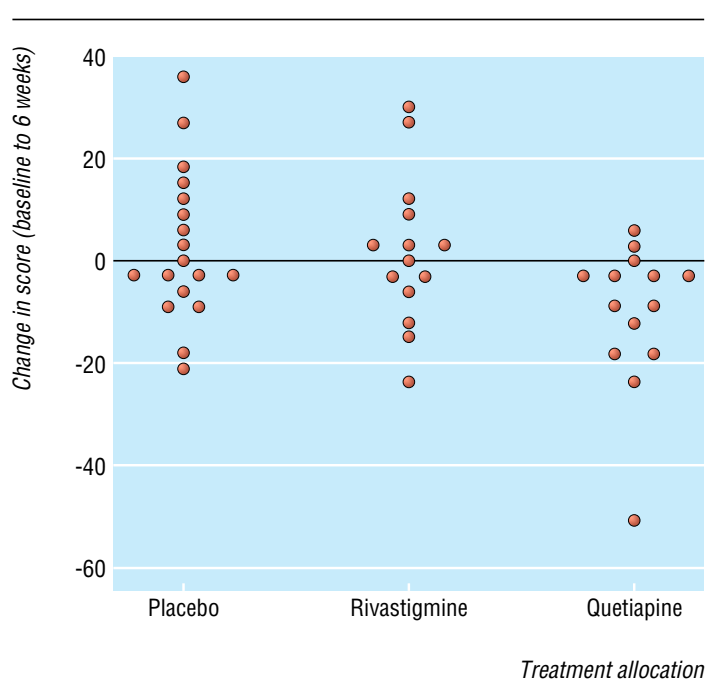

Fig 3 Change in score on severe impairment battery (baseline to six weeks) by treatment group indicated to determine the potential value of central cholinesterase inhibitors on cognition and behaviour in people with severe dementia and clinically significant agitation.

\section{Strengths and limitations}

Patients in the placebo group had an average improvement of 6.2 points on their agitation inventory score and an average improvement of 3.2 points on their severe impairment battery over six weeks. This improvement is consistent with most previous reports. ${ }^{3}$ Trials focusing on cognition often report improvements in placebo groups over three to four months, although they do not usually persist for as long as 26 weeks. The modest improvement we observed is probably explained by a Hawthorne effect-namely, residents in an unstimulating environment ${ }^{18}$ respond positively to the increased interaction as part of the study procedures. Though our study is limited because of the modest sample size, multiple evaluations, and the substantial proportion of patients who were unable to complete the severe impairment battery, the possible impact of quetiapine on cognition is clinically important.

We did not adjust for multiple significance testing because we considered the six week results would be the most important because of the anticipated dropout rate; comparison of treatment versus placebo was of primary importance, hence the interpretation of the significant result; and comparison of active treatments was always going to be of secondary importance, hence the caution with the six week result for rivastigmine versus quetiapine. When interpreting the cognitive function result for quetiapine compared with placebo, it can be argued that, in the light of a lack of evidence of efficacy, even a suggestion of a decline in what was a secondary outcome is noteworthy. As one could argue that it is not necessary to show significant evidence of harm under these particular circumstances, a formal adjustment was not deemed necessary.

The severe impairment battery has good concurrent validity and is sensitive to longitudinal change ${ }^{19}$ in people with severe dementia. In addition, it has been used in several placebo controlled trials of central cholinesterase inhibitors ${ }^{20}$ or memantine $^{21}$ in people with severe dementia, showing enough sensitivity to identify benefits of treatment. It is, therefore, a robust measure of cognitive change in these patients.

\section{Conclusion}

Given current concerns about the risk of stroke with risperidone and olanzapine, ${ }^{4}$ further studies are required to enable evidence based pharmacological management of behavioural disturbance in dementia. Quetiapine and rivastigmine seemed of no benefit in patients with dementia and agitation in institutional care, and quetiapine was associated with greater cognitive decline than placebo. Our results suggest that quetiapine should not be used as an alternative treatment to risperidone or olanzapine in people with dementia and highlight concerns regarding the long term use of antipsychotics in these patients.

We thank the Alzheimer's Research Trust for their support in our programme of work to evaluate the impact of antipsychotics on cognition; Alistair Burns for his helpful comments on the manuscript; and Andrea Burton for her helpful advice regarding the sensitivity analysis.

Contributors: $\mathrm{CB}, \mathrm{EJ}, \mathrm{MM}-\mathrm{L}$, and RJ designed the study. $\mathrm{CB}$ supervised implementation of the study overall, and EJ designed the analysis. CB, MM-L, and LL were responsible for day to day supervision of study implementation. SD, AE, SS, CM, LL, CBan, and RE collected the data. AS, AT, and JO'B formed the data monitoring committee. CB, MM-L, RJ, SD, AT, JO'B, CBan, and RJ wrote the manuscript. RJ is guarantor.

Funding: The study was funded largely from general donations to CB's research programme and profits from previously completed commercially 


\section{What is already known on this topic}

Antipsychotic drugs are widely used for the control of behavioural manifestations in dementia, of which agitation is the most common

Central cholinesterase inhibitors are increasingly used to treat the behavioural manifestations of dementia

Observational data have suggested that (typical) antipsychotics might accelerate cognitive decline in dementia

\section{What this study adds}

Central cholinesterase inhibitors and atypical antipsychotics are not effective for the treatment of agitation in people with dementia

Quetiapine (an atypical antipsychotic) is associated with accelerated cognitive decline

funded clinical trials, with additional support from the Alzheimer's Research Trust.

Competing interests: C Ballard has received honorariums and research donations to support his general research programme from Astra Zeneca and Novartis.

Ethical approval: The study was approved by a properly constituted local research ethics committee.

1 Ballard C, Holmes C, McKeith I, Neill D, Lantos P, Cairns N, et al. Psychiatric morbidity in dementia with Lewy bodies: a prospective clinical and neuropathological comparative study with Alzheimer's disease. Am J Psychiatry 1999;156:1039-45.

2 McGrath AM, Jackson GA. Survey of prescribing in residents of nursing homes in Glasgow. BMJ 1996;314:611-2. 3 Ballard C, O'Brien J. Treating behavioural and psychological signs in Alzheimer's dis-

4 Committee on Safety of Medicines. Atypical antipsychotic drugs and stroke. www.mca.gov.uk (accessed 9 March 2004).

5 Tariot P. Atypical antipsychotics for the treatment of BPSD. Int Psychogeriatr (in press).

6 Cummings JL. Use of cholinesterase inhibitors in clinical practice: evidence-based recommendations. Am J Psychiatry 2003;11:131-45.

7 McShane R, Keene J, Gedling K, Fairburn C, Jacoby R, Hope T. Do neuroleptic drugs hasten cognitive decline in dementia? Prospective study with necropsy follow up. BMJ 1997;314:266-70.

8 Cohen-Mansfield J. Assessment of disruptive behaviour/agitation in the elderly: function, methods, and difficulties. J Geriatr Psychiatry Neurol 1995;8:52-60.

9 McKhann G, Drachman D, Folstein M. Clinical diagnosis of Alzheimer's disease: report of the NINCDS-ADRDA work group under the auspices of Department of Health and of the NINCDS-ADRDA work group under the auspices of Department of Hea
Human Services task forces on Alzheimer's disease. Neurology 1984;34:939-44.
10 Saxton J, McGonigle-Gibson K, Swihart A, Miller M, Boller F. Assessment of severely impaired patients: description and validation of a new neuropsychological test battery. Psychol Assess 1990;2:298-303.

11 Reisberg B. Functional assessment staging (FAST). Psychopharmacol Bull 1988;24:653-9

12 Scharre DW. Chang SI. Cognitive and behavioral effects of quetiapine in Alzheimer disease patients. Alzheimer Dis Assoc Disord 2002;16:128-30.

13 Tariot PN, Erb R, Podgorski CA, Cox C, Patel S, Jakimovich L, Irvine C. Efficacy and tolerability of carbamazepine for agitation and aggression in dementia. Am J Psychiatry 1998:155:54-61

14 Coffey ET, Akerman KE, Courtney MJ. Brain derived neurotrophic factor induces a rapid upregulation of synaptophysin and tau proteins via the neurotrophin receptor TrkB in rat cerebellar granule cells. Neurosci Lett 1997;227:177-80.

15 Perry EK, Kilford L, Lees AJ, Burn DJ, Perry RH. Increased Alzheimer pathology in Parkinson's disease related to antimuscarinic drugs. Ann Neurol 2003;54:235-8.

16 Richelson E. Receptor pharmacology of neuroleptics: relation to clinical effects. J Clin Psychiatry 1999;10;5-14.

17 Fleming K, Thyrum P, Yeh C, Vargo DL, Potkin SG. Cognitive improvements in psychotic subjects treated with "Seroquel" (quetiapine fumarate): an exploratory study. JClin Psychopharmacol 2001:21:527-9.

18 Ballard C, Fossey J, Chitramohan R, Howard R, Burns A, Thompson P, et al. Quality of care in private sector and NHS facilities for people with dementia: a cross-sectional survey $B M J$ 2001;323:426-7.

19 Schmitt FA, Ashford W, Ernesto C, Saxton J, Schneider LS, Clark CM, et al. The severe impairment battery: concurrent validity and the assessment of longitudinal change in Alzheimer's disease. The Alzheimer's disease cooperative study. Alzheimer Dis Assoc Disord 1997;11(suppl 2):S51-6.

20 Feldman H, Gauthier S, Hecker J, Vellas B, Subbiah P, Whalen E, et al. A 24-week, randomized, double-blind study of donepezil in moderate to severe Alzheimer's disease Neurology 2001;57:613-20.

21 Reisberg B, Doody R, Stoffler A, Schmitt F, Ferris S, Mobius HJ. Memantine study group. Memantine in moderate-to-severe Alzheimer's disease. $N$ Engl $J$ Med 2003;348:1333-41

doi $10.1136 / \mathrm{bmj} .38369 .459988 .8 \mathrm{~F}$

Institute of Psychiatry, King's College, London SE5 8AF

Clive Ballard professor of age related diseases

Marisa Margallo-Lana senior lecturer in old age psychiatry

Centre for Statistics in Medicine, Oxford University, Oxford OX3 7LF

Edmund Juszczak senior medical statistician

University of Newcastle, Institute of Ageing, Newcastle General Hospital, Newcastle upon Tyne NE4 4BE

Simon Douglas research nurse

Alan Swann consultant in old age psychiatry

Alan Thomas consultant in old age psychiatry

John O'Brien professor of old age psychiatry

Anna Everratt psychology assistant

Stuart Sadler psychology assistant

Clare Maddison psychology assistant

Lesley Lee research nurse

Ruth Elvish psychology assistant

Oxfordshire Mental Healthcare NHS Trust, Fiennes Unit, Horton Hospital, Banbury OX16 9BF

Carol Bannister consultant in old age psychiatry

Oxford University Department of Psychiatry, Warneford Hospital, Oxford OX3 7JX

Robin Jacoby professor of old age psychiatry

Correspondence to: R Jacoby Robin.Jacoby@psych.ox.ac.uk 